\title{
A Critical View on Environmental and Human Risk Acceptance Criteria
}

\author{
Dimitris Diamantidis
}

\begin{abstract}
Natural and technological hazards have a major impact on environmental and human safety. Risk assessment plays an essential role in the risk management of the effects of such hazards. This contribution summarizes first the general formulation of risk depending on two parameters: hazard probability and associated consequences. Problems faced in industrial applications based on the experience of the author are discussed. Risk acceptance criteria for the contributors to risk i.e. human, economic and environment are critically reviewed. Resilience based criteria are also included and conclusions for future developments are provided.
\end{abstract}

Index Terms-Environmental risk, human risk, risk acceptance, risk analysis, safety measures.

\section{INTRODUCTION}

During the last decades risk analysis methods have gained increasing acceptance in many industrial fields, as for example in the oil, chemical and transportation industry. General consensus has been reached related to the definition of risk, but when it comes to a decision regarding risk acceptance and selection of safety measures various methods and criteria are used.

One reason for that is that risk includes a considerable number of influencing factors, for example human risk perception is dependent on factors such voluntary or involuntary, ordinary or catastrophic, natural or man-made risk etc. In addition the consequences which affect the risk concern human losses, economic impact and environmental damage which need to be quantified and considered together. Research activities on risk perception are therefore of great importance to support risk assessment and decision-making.

This contribution addresses the aforementioned aspects by reviewing existing methodologies and by providing recommendation for future developments. The relevant definitions for the average reader are provided first. The risk analysis methodology is summarized and experience of the author from various risk studies is reflected. Risk and safety acceptance criteria in the literature and reflected in codes and standards are critically presented both for human safety and environmental protection. The use and possible combination of such criteria are discussed based on the experience of the author in various studies. The connection to the resilience concepts is thereby considered. Decision criteria based on cost optimization are included and illustrated in characteristic

Manuscript received October 9, 2015; revised April 9, 2016. This work has been supported by the Regensburg Centre of Energy and Resources (RCER) of the Ostbayerische Technische Hochschule Regensburg (OTH Regensburg) and the Technology- and Science Network Oberpfalz (TWO).

Dimitris Diamantidis is with the Ostbayerische Technische Hochschule Regensburg, Germany (e-mail: Dimitris.diamantidis@ oth-regensburg.de). examples. Recommendations for future developments especially in standards are drawn.

\section{DEFINITIONS}

The following definitions are provided in order to develop a consistent understanding of selected terms within the scope of the study. They are based mainly on the document [1].

Acceptable Risk: A level of risk, which is generally not perceived to be serious by an individual or society, and which may be considered as a reference point in criteria related to risk.

Accident: Undesired event leading to a loss.

Consequence: A possible outcome of an accident.

Environmental damage: A direct or indirect reduction of one or several resources resulting from the undesired event.

Hazard: A set of circumstances with a potential for causing events with undesirable consequences.

Hazard Scenario: A sequence of possible events related to a given hazard leading to undesired consequences.

Probability: The likelihood or degree of certainty of a particular event occurring during a specified period of time.

Risk: A measure of the danger that undesired events represent for humans, environment, or economic infrastructure. The risk is expressed as the probability multiplied by the consequences of undesired events.

Risk Assessment: The process of risk analysis, risk acceptance and option analysis (safety measure evaluation).

Safety: The state of being protected against the adverse consequences of a hazard.

Hazards and related undesired (accidental) events can be classified into:

1) anthropogenic hazards such as for example fires, mining accidents, transportation accidents, terrorist attacks;

2) accidents triggered by natural hazards such as for example floods, storms, earthquakes, drought, landslides.

\section{RISK ANALYSIS}

Risk analysis procedures have been well established in the past two decades and have been successfully applied to various industrial fields such as to the oil or the transportation industry. Background information can be found among other in [2]-[5]. The definition of risk is based on the selection of appropriate hazard scenarios $H_{i}$ which may lead to accidental events $E_{i j}$ (e.g. overtopping of dams, rupture of pipelines) with adverse consequences $C_{i j}$. The consequences of adverse events $E_{i j}$ may consist of several components denoted as $C_{i j, k}$ including human losses (fatalities, injuries), economic consequences (replacement/repair, clean-up costs, business 
interruption etc.) and environmental damage (for example pollution in water, groundwater etc.) The components $R_{k}$ of the total risk may be assessed from the relationship

$$
R_{k}=\sum_{i, j} C_{i j, k} P\left(E_{i, j} / H_{i}\right) P\left(H_{i}\right)
$$

The components reflect human, economic and environmental risk. If an acceptable risk $R_{A}$ is specified, the system under study can be assessed for each risk component on the basis $R_{k}<R_{A}$. The total risk is also considered for decision purposes. The guidance for determination of the criterion of acceptable risks $R_{A}$ is discussed in the next sections. When the criterion of acceptable risks is not fulfilled, it is necessary to modify the system by appropriate interventions aiming at reducing probability of occurrence of adverse events (protection measures) or at reducing consequences (mitigation measures). There is a number of difficulties faced in the risk analysis procedures, such as:

1) Calculation of small probabilities frequently of the order of $10^{-5}$ per year or less and many times based on limited data available;

2) Computation of consequences for the various considered scenarios based on models and input parameters derived from previous experience;

3) Combination of consequences to assess the total risk from its contributors (human, economic, environmental).

The results of a risk analysis are associated to uncertainties, which can be attributed consequently to the relevance and significance of the data basis, to the applied calculation models and also to possible assumptions, premises and expert judgement used. Uncertainties can be dealt with by performing sensibility analyses or better by using probabilistic risk analysis techniques. In the last procedure the probability of exceeding a specified level of risk (in term losses per year) can be calculated and used for decisions.

\section{RISK ACCEPTANCE}

\section{A. Human Safety}

The nature of risk determines its acceptability which is associated with several properties of it and related factors such as: voluntary vs. involuntary, controllability vs. uncontrollability, familiarity vs. unfamiliarity, direct/indirect consequences, existence of alternatives, type and nature of consequences, derived benefits, communication in the media, information availability, personal involvement, memory of consequences, degree of trust in standards and regulatory bodies. When people are familiar with risk involved in an activity they are more willing to accept it. Type and nature of consequences are another important property of risk, since risks due to events causing more damage and fatalities (for example nuclear power plant accidents) are more difficult to accept. Derived benefits of society and the individual play a significant role in risk acceptance. The risk acceptance depends also on the level of available information, personnel involvement, memory of consequences and degree of trust in regulatory bodies. The risk communication plays thereby an important role and should be always highlighted.

In general two types of human risk are distinguished: the individual and the societal risk. The annual probability of being harmed describes the risk to an individual due to a hazardous situation and is called the individual risk. In this contribution the risk to society as a whole is of prime interest and therefore this societal risk is considered herein.

The societal risk is often represented in the form of a numerical F-N-curve. This F-N-curve ( $\mathrm{N}$ represents the number of fatalities, $\mathrm{F}$ the frequency of accidents with more than $\mathrm{N}$ fatalities) shows the probability of exceedance as a function of the number of fatalities $\mathrm{N}$ on a double logarithmic scale [4]:

$$
1-F_{N}(x)=P(N>x)=\int_{x}^{\infty} f_{N}(x) d x
$$

In which $f_{N}(x)$ is the probability density function of number of fatalities per year; $F_{N}(x)$ the probability distribution function of the number of fatalities per year, representing the probability of less than $\mathrm{x}$ fatalities per year.

A simple measure for societal risk is the annual expected value of the number of fatalities $E(N)$ :

$$
E(x)=\int_{0}^{\infty} x f_{N}(x) d x
$$

The expected risk value is frequently used to compare alternative projects in terms of their inherent risk.

Based on the F-N curves the so-called ALARP (as low as reasonably possible) region can be defined as exemplified in Fig. 1. The upper limit represents the risk that can be tolerated in any circumstances while below the lower limit the risk is of no practical interest. Such acceptability curves have been developed for various industrial fields including the chemical and the transportation industry [2], [5].

F-N curves were originally developed for nuclear hazards to illustrate thresholds that reflect societal aversion to multiple fatalities during a single catastrophic event. The graph as shown in Fig. 1 is subdivided into four areas: unacceptable risk; tolerable risk that should be reduced further if practicable according to the as low as reasonably practicable (ALARP) principle; broadly acceptable risk; and a region of low probability but with the potential for $>1000$ fatalities that requires intense scrutiny.

Typical F-N curves reported in the literature show different curves for the same industrial activity in various countries or for different industrial activities in the same country. The following general formula has been proposed to represent the societal human risk acceptance criterion:

$$
F \leq a N^{-k}
$$

In which $a$ and $k$ are preset constants. It can berelated to statistical observations from natural and man-made hazards. Some natural hazards show relationships with $k$ slightly smaller than unity while most manmade hazards are described by a relationship with $k>1$. From statistical observations the constants $a$ and $k$ vary widely depending on the type of hazard and the type of technical activity. It has been proposed to set the constants such that the curve envelops the curves for most natural hazards and some more common man made hazards from below, see [6]. For acceptable risks in case of structural failures as an example the constant would be around $a=10^{-6}$ and for marginally acceptable risks $a=10^{-4} ; k=1$ represents risk-neutral curves, 
$k>1$ describes curves with risk aversion and $k<1$ curves with risk proneness. The case of $k<1$ leads to infinitely large expected losses (in terms of lives or cost) and, therefore, is not acceptable.

In the ALARP principle the "width" between upper and lower bound curves is of importance. In many cases this width is two orders of magnitudes allowing for too much flexibility in practical cases.

It is noted further here that human safety does not only involve fatalities but also injuries. In many studies injuries are related to fatalities by using a multiplicative factor as for example 0.1 for moderate injury and 0.5 for major injury. Based on this simple procedure weighted fatalities can be obtained.

The aforementioned societal human criteria can be also represented in a so-called risk-matrix. For that purpose qualitative hazard probability levels suitable for use in assessment of risks are used together with hazard severity levels of accidental consequences. The hazard probability levels and the hazard severity levels can be combined to generate a risk classification matrix. The authority is usually responsible for defining the tolerability of the risk combinations contained within the risk classification matrix. The risk matrix approach is relatively insensitive to uncertainty as the classification of categories is relatively coarse. It is easy to be used and interpreted however it has a low level of precision. In case of risk of road tunnels a risk acceptability matrix is proposed in [5] and has been used to verify risks in existing road tunnels.

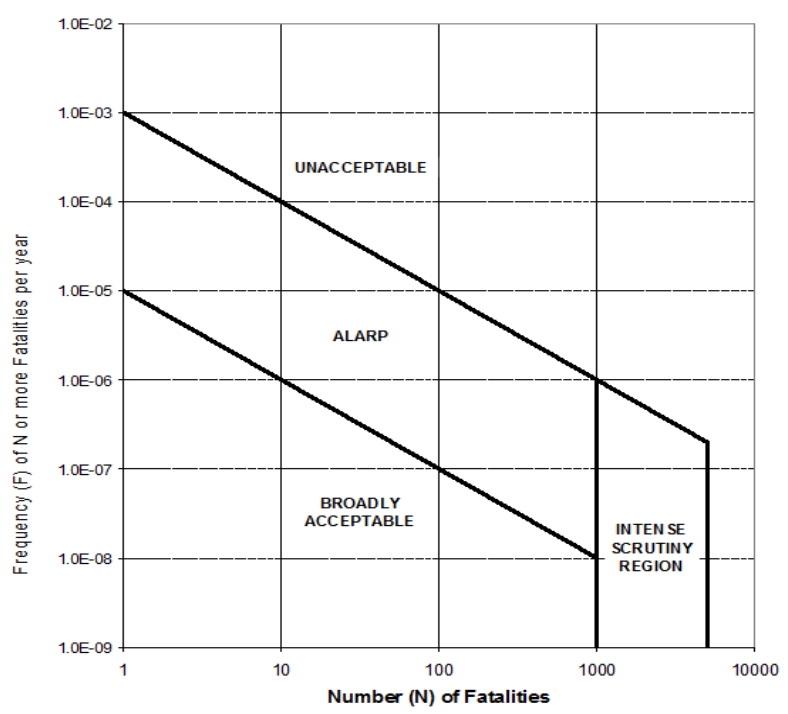

Fig. 1. Illustration of the ALARP principle (example).

\section{B. Economic Risk}

Besides human safety economic risk plays an important role in decision making. Economic consequences are direct consequences related for example to repair of initial damage and replacement of equipment/contents and indirect consequences such as loss of production, temporary relocation, rescue costs, loss of reputation. Similarly to F-N curves F-D curves can be derived related to the economic damage $D$ as follows [4]:

$$
\begin{gathered}
1-F_{D}(x)=P(D>x)=\int_{x}^{\infty} f_{D}(x) d x \\
E(D)=\int_{0}^{\infty} x f_{D}(x) d x
\end{gathered}
$$

In which $\left(f_{D}(x)\right)$ is the density function and $F_{D}(x)$ is the probability distribution function of the economic damage and $E(D)$ is the expected value of the economic damage.

Analogous to the F-N curve and the expected number of fatalities it can be shown that the area below the F-D curve is equal to the expected damage and reflects the expected risk value. It is noted that economic damage includes various contributors such as repair/replacement costs, loss of production, loss of business, compensations, loss of reputation, changes in professional practice which need to be considered and added; this task itself is not always straightforward and is associated to uncertainties as mentioned before.

It is further emphasized that economic damage is directly related to monetary values: the dimension of the risk is expected loss in currency per year.

\section{Environmental Risk}

Main focus is given herein to the environmental risk criteria which can be defined in a similar way. The environmental consequences can be presented in terms of permanent or long-term damage to terrestrial, freshwater, marine habitats and groundwater reservoir. Thereby the parameter of damage can be the damaged area. A different parameter has been selected by NORSOK [7] in which the recovery time from the accident defines the damage. The overall principle implies that recovery following environmental damage shall have an insignificant duration when compared to the expected period (return period) between such damages.

NORSOK (the competitive standing of the Norwegian offshore sector) has proposed the probability of exceedance of the time $T$ needed by the ecosystem to recover from the damage as a measure for environmental risk:

$$
1-F_{T}(x)=P(T>x)=\int_{x}^{\infty} f_{T}(x) d x
$$

where $F_{T}(x)$ is probability distribution function of the recovery time; $f_{T}(x)$ probability density function of the recovery time of the ecosystem, see also [4].

Table I presents acceptable frequency limits based on an insignificant 5\% value. Fig. 2 and Fig. 3 illustrate graphically the criteria of Table I in order to show the compatibility with the human and economic criteria. Fig. 2 shows the target return period of the accident as a function of the recovery time, an event with 100 years return period is associated to a recovery time of 5 years. Fig. 3 shows analogously to the F-N diagram the corresponding F-T curves, with $T$ the recovery time.

TABLE I: ACCEPTANCE CRITERIA ENVIRONMENTAL RISK [7]

\begin{tabular}{lll}
\hline \hline $\begin{array}{l}\text { Environmental } \\
\text { damagecategory }\end{array}$ & $\begin{array}{l}\text { "Average" } \\
\text { recovery }\end{array}$ & Acceptable frequency limit \\
\hline Minor & $1 / 2$ years & $<1$ event per 10 years \\
Moderate & 2 years & $<1$ event per 40 years \\
Significant & 5 years & $<1$ event per 100 years \\
Serious & 20 years & $<1$ event per 400 years \\
\hline \hline
\end{tabular}

The compatibility to the human and economic risk criteria is obvious. However compared to the human safety criteria 
the related return periods are in lower ranges or the corresponding annual frequency in higher ranges. The recovery time is again a parameter associated to considerable uncertainties and includes parameters such as resources of interventions, rapidity of interventions etc. Recovery time reflects more the parameter of resilience which is directly defined as a function of the recovery time.

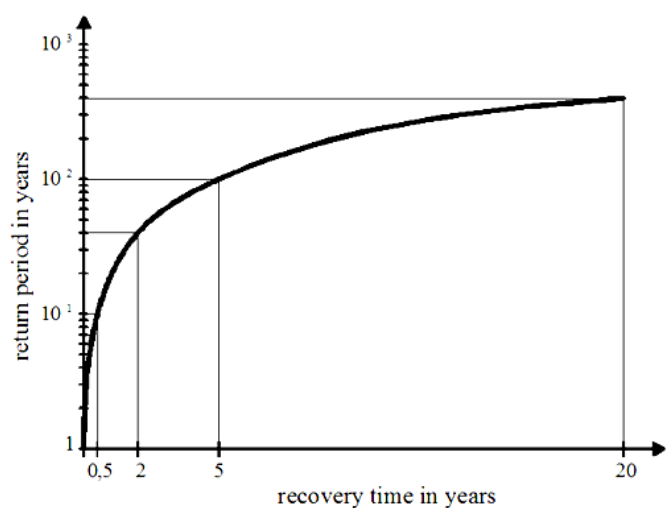

Fig. 2. Acceptable accident return period versus recovery time for the values of Table I based on [7]

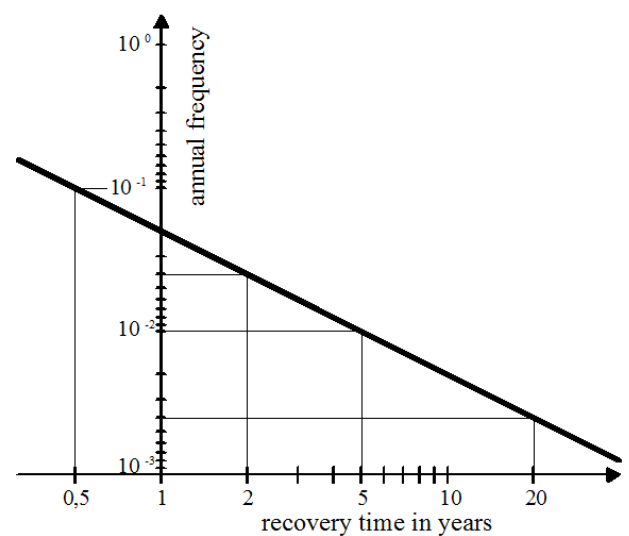

Fig. 3. Acceptable annual frequency versus recovery time for the values of Table I based on [7].

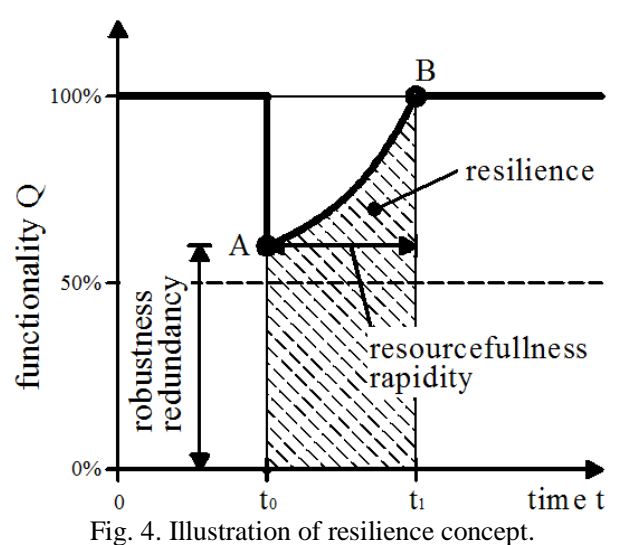

Resilience reflects the capability to sustain a level of functionality or performance for a given system over a period defined as the control time. Resilience concepts are critically reviewed among other in [8], [9]. Resilience is the ability of a system to continue providing some basic functionality when exposed to some external extreme accidental action and / or to recover quickly from the effects of such an accident as shown in Fig. 4 (from point A to point B). It depends on robustness, redundancy during the accident and on resourcefulness and rapidity during recovery. Consequently it is a complex construct involving parameters which may be organizational; societal or technological in nature. Such parameters affect also the recovery time from an environmental damage.

The concept of resilience as discussed in [8], [9] and illustrated in a simple form in Fig. 4 has been used in many studies to define a resilience index:

$$
r=\frac{\int_{t_{0}}^{t_{1}} Q(t) d t}{100\left(t_{1}-t_{0}\right)}
$$

where $Q$ is the infrastructure functionality, or the performance of a system, $t_{0}$ is the time of incident or disturbance occurrence, and $t_{1}$ is the time to full recovery. According to this model, the units of resilience are performance per unit time, where performance can be measured in percent of full performance. The connection to the recovery time in case of environmental risk becomes obvious. However the concept of resilience is used in general for functionality of systems of infrastructures by considering all related parameters. Further efforts are needed to include the concept of resilience in risk assessment.

\section{DECISION CRITERIA}

The problem of identifying an acceptable level of safety can also be formulated as an economic decision problem by combining all types of risk. Economic value should be thereby addressed to social acceptance of human and environmental losses. In case of environmental damage total costs per damaged unit area can be defined. In case of human losses the monetary valuation of human life is a difficult and for many an unethical task.

A utility based approach by using the Life Quality Index (LQI) has been developed in [10], and used also in recent standard development [11] and related background work [12].

The strategy is based on a social indicator that describes the quality of life as a function of the gross domestic product, life expectation and the life working time. The LQI is a compound societal indicator, which is defined as a monotonously increasing function of two societal indicators: the gross domestic product per person per year $g$, and the life expectancy at birth $e$ as follows:

$$
L Q I=g^{w} e^{1-w}
$$

The exponent $w$ is the proportion of life spent in economic activity. In developed countries for example it is assumed to $w$ app. 1/8. Using this Life Quality Index Criterion, the optimum acceptable Implied Cost of Averting a Fatality (ICAF) can then be derived.Other similar parameters reflecting the monetary value, which a society should be willing to invest for saving a human life (societal willingness to pay)may be used.

By applying the safety versus cost-benefit approach, risk acceptability criteria have been implemented in industrial projects by evaluating the costs and benefits of each possible investment into safety. For each possible safety measure, $k$, the following parameters are therefore considered:

$C_{I k}$ : investment costs

$C_{A k \text { : }}$ annual maintenance/operation costs 
$T_{L}$ : desired lifetime of potential safety measure

$d R_{k}$ : risk reduction due to implementation of the safety measure $k$, divided into:

$d R_{H k}:$ reduction related to human risk

$d R_{F k}$ : reduction related to economic (financial) risk

$d A_{E k}$ : reduction of damaged area of the environment

$c_{u}$ : unit costs to recover the damaged area

In addition, if we consider a discount rate $\delta\left(T_{L}\right)$ the evaluation of each individual safety measure can be made on the basis of the aforementioned assumptions related to risk acceptability, cost functions, and risk reduction, using the following inequality:

$$
\left(C_{I k} \delta\left(T_{L}\right)\right) / T_{L}+C_{A k}<I C A F d R_{H k}+d R_{F k}+c_{u} d A_{E k}
$$

It is recommended that life-saving costs must be discounted at the same rate as other investments to avoid inconsistency. If the inequality (9) is satisfied (i.e. the cost of the safety measure is less than the value of the benefits obtained) then the safety measure is beneficial. If an overall budget $B$ is available for annual safety investments the optimal package of safety measures can be determined by

1) considering the most beneficial of the aforementioned procedures for ranking of each safety measure;

2) taking into account dependencies of measures (correlations) by approximations;

3) optimizing for a fixed $B$ in such a way that total risk reduction is maximized.

The concept of the LQI has been recently further developed within the work of standards, see for example [11]. Further aspects are analytically described in [12]. A simple example is presented to illustrate the application of inequality (9). One should however bear in mind that many safety measures are related to new technological developments and their influence on risk reduction cannot be assessed on the basis of data but only on the basis of judgement, expert opinion and simulation of accident scenarios development. Again all uncertainties should be taken into account either by sensitivity analyses or by probabilistic analyses i.e. by considering probabilities that costs exceed benefits.

Example:

The implementation of a protective wall against flood at a river as a safety measure should be verified. The parameters entering the inequality (9) have been considered for example as:

$$
\begin{aligned}
& C_{I k}=1000000 \mathrm{USD} \\
& C_{A k}=50000 \mathrm{USD} \\
& T_{L}=20 \text { years and } \delta\left(T_{L}\right)=1.01^{20}(1 \% \text { rate }) \\
& d R_{H k}=2 \times 10^{-2} \text { fatalities per year } \\
& d R_{F k}=30000 \mathrm{USD} \\
& I C A F=3000000 \mathrm{USD} \\
& d A_{E k}=1000 \mathrm{~m}^{2} \\
& c_{u}=50 \mathrm{USD}^{2} \text { per } \mathrm{m}^{2}
\end{aligned}
$$

By implementing inequality (9) it follows that the considered safety measure is beneficial under the considered criteria.

\section{SUMMARY AND CONCLUSIONS}

The parameters and dimensions of risk acceptance criteria have been reviewed in this paper. The general formulation of risk depending on two parameters: hazard probability and associated consequences has been discussed after providing useful definitions. Problems faced in industrial applications based on the experience of the author have been discussed. Risk acceptance criteria for the contributors to risk i.e. human, economic and environment have been critically reviewed. Resilience based criteria have been also included and conclusions for future developments are provided. Related to the selection of acceptability criteria the following procedures are frequently applied:

1) the current activity in one sector or country is considered as acceptable and used to derive acceptance criteria for another sector;

2) the social acceptance is also based on discussions with operators and/or authorities;

3) the safety measures are selected on the basis of on cost optimization.

In assessing risk the uncertainties of the influencing parameters is of major importance and should be dealt with. Aspects for future developments could include:

1) analysis of metrics to quantify better environmental damage based on a survey of experienced disasters,

2) sophisticated implementation of resilience considerations in the criteria,

3) combination procedures for considering the overall risk acceptance contributors,

4) determination of consistent and uniform criteria in standards and

5) practical recommendations regarding the treatment of uncertainties.

\section{REFERENCES}

[1] CIB TG 32, Risk Assessment and Risk Communication in Civil Engineering, Rotterdam: CIB, 2001.

[2] Reducing Risks Protecting People, Norwich (UK): Health and Safety Executive, 2001.

[3] M. G. Stewart and R. E. Melchers, Probabilistic Risk Assessment of Engineering Systems, Berlin: Springer, 1997.

[4] S. N. Jankman, "An overview of quantitative risk measures for loss of life and economic damage," J. of Hazardous Materials, pp. 1-30, 2003.

[5] D. Diamantidis and M. Holický, "Risk and robustness of road tunnels," in Proc. ICASP Conference, Zürich, Switzerland, August 2011.

[6] D. Diamantidis, Probabilistic Assessment of Existing Structures, Joint Committee on Structural Safety, RILEM Publications, 2001.

[7] Norsok Standard, Risk and Emergency Preparedness Analysis. Annex c. Methodology for Establishment and Use of Environmental Risk Acceptance Criteria, 1998.

[8] A. Caverzan and G. Solomos, Review on Resilience in Literature and Standards for Critical Built-Infrastructure, Joint Research Center, European Commission, 2014.

[9] B. M. Ayyub, Systems Resilience for Multihazard Environments. Definition, Metrics, and Valuation for Decision Making.

[10] J. S. Nathwani, "Affordable safety by choice: The life quality method," Institute for Risk Research, University of Waterloo, Canada, 1997.

[11] ISO 2394, General Principals on Reliability of Structures, 2014.

[12] R. Rackwitz, "Optimization - The basis of code-making and reliability verification," Structural Safety, vol. 22, pp. 27-60, 2000.

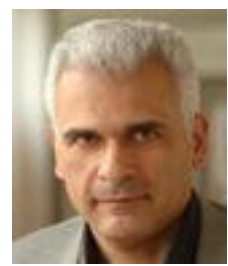

Dimitris Diamantidis is a professor at the Ostbayerische Technische Hochschule Regensburg in Germany. He holds a diploma and a doctorate degree from the Technical University of Munich in Germany and has working experience with Det Norske Veritas in Oslo, Norway and with D' Appolonia in Genova, Italy. He is a consulting engineer for projects related to risk assessment $\&$ an author of about 100 publications. 